\title{
Innovación en las pymes industriales: una visión desde el modelo interactivo
}

\author{
Eneka Albizu, Mikel Olazaran, Beatriz Otero y Cristina Lavía \\ Universidad del País Vasco (UPV-EHU) \\ eneka.albizu@ehu.es; mikel.olazaran@ehu.es; \\ beatriz.otero@ehu.es; cristina.lavia@ehu.es
}

Resumen: Esta investigación analiza cómo se producen las innovaciones en las pymes industriales tomando como marco conceptual el «modelo interactivo» de innovación propugnado por la Escuela de Aalborg. El trabajo de campo, efectuado en Guipúzcoa, cuenta con una fase cualitativa (47 entrevistas en profundidad) y otra cuantitativa (encuesta telefónica a 147 empresas). Los resultados obtenidos sugieren que las pymes industriales analizadas innovan de acuerdo con el modelo DUI (doing, using, interacting) frente al modelo STI (science, technology, innovation).

Palabras clave: innovación, pyme, DUI, SRI.

Abstract: This paper looks at innovation processes in industrial SMEs from the point of view of so-called «interactive learning» or Lundvall's Aalborg model. The paper is based on both qualitative (47 semi-structured interviews) and quantitative (phone survey of 147 firms) empirical fieldwork carried out in the Basque province of Guipuzcoa (Northern Spain). The empirical evidence shows that industrial SMEs from that region innovate in accordance with the "DUI mode» (doing, using, interacting), and that they are far from the «STI mode» (science, technology, innovation).

Keywords: innovation, pyme, DUI, SRI. 


\section{Introducción}

En los últimos años, y más aún en un contexto de grave crisis económica unida —en el caso español- a una crisis más profunda del modelo productivo, surgen con fuerza los discursos sobre la innovación como bandera del cambio. Pero, a pesar de ello, no parecen estar tan claras las respuestas a preguntas como ¿qué es la innovación?, ¿son las pymes (organizaciones que forman la mayor parte del tejido empresarial español) innovadoras?, ¿`cómo son los procesos de innovación en estas compañías?, ¿hasta qué punto la proximidad (espacial, social) y la existencia de estructuras de I+D (como los centros tecnológicos) facilitan la innovación en las pymes?

En este trabajo pretendemos responder a estas preguntas basándonos en un doble estudio empírico (cualitativo y cuantitativo) sobre pymes industriales realizado en el País Vasco.

A nuestro entender, en los discursos sociales sobre la innovación, junto a problemas reales circulan tópicos que es necesario matizar. Veamos un ejemplo:

Históricamente España no ha sido un país innovador [... Uno de los grandes agujeros negros de las empresas de nuestro país son los paupérrimos resultados de $\mathrm{I}+\mathrm{D}$, muy especialmente en pymes [...] Las partidas destinadas a innovar en muchas ocasiones [... ] acaban aplicando modelos gestados y validados internacionalmente para aplicarlos al mercado local. Nos limitamos a importar tecnología y clonar ideas del exterior 12 o 24 meses más tarde... [Hay] poca inversión en $\mathrm{I}+\mathrm{D}$ y en las raras ocasiones en que ésta existe (como en el caso de algunas universidades españolas), queda limitada a un ejercicio teórico sin vocación de futuro comercial [...] (Suárez, 2009).

Uno de los resultados de nuestro estudio, como veremos más adelante, es que las pymes industriales innovan, pero el modo en que lo hacen está alejado de lo que se consideran actividades de $\mathrm{I}+\mathrm{D}$ en sentido estricto (importancia del conocimiento codificado, del personal de I+D con educación terciaria, de las relaciones con instituciones científicas o tecnológicas, de las patentes, etc.). Las pymes industriales están inmersas en procesos de búsqueda y creación de conocimiento (captación, incorporación y desarrollo de nuevos componentes tecnológicos, resolución de problemas, imitación de competencias y rutinas externas, nuevas combinaciones de conocimiento, etc.). Se trata de una innovación incremental donde predomina el conocimiento tácito. Pero esto es innovación y, en contra de lo que pudiera parecer en la cita anterior, no resulta nada fácil llevarla a cabo. 
Nuestro marco conceptual se sitúa dentro de la perspectiva de los sistemas nacionales de innovación, y más en concreto en el «modelo interactivo» de innovación estudiado por Lundvall y colaboradores en el caso danés. La perspectiva del sistema nacional de innovación surgió con fuerza en los años noventa del pasado siglo, poniendo en cuestión los postulados ortodoxos sobre el papel del conocimiento como genérico, codificable, accesible sin coste e independiente del contexto (Freeman, 1987; Dosi et alii, 1988; Lundvall, 1992a; Nelson, 1993; Edquist, 1997). Esta perspectiva estudia la innovación como fenómeno sistémico, donde se interrelacionan actores y factores tanto internos como externos a la empresa, dentro de un entorno institucional y cultural. El cambio técnico sería un proceso endógeno, dependiente de una trayectoria y condicionado por un contexto organizacional, institucional y cultural.

Con su noción de interactive learning, Lundvall y equipo han profundizado en el carácter interactivo de los procesos de innovación, que son el resultado, por un lado, de la interacción continua entre agentes internos a la empresa (por ejemplo, entre diseño, producción y marketing, entre ingenieros y trabajadores de producción, o entre personal de la Oficina Técnica y el Servicio de Asistencia Técnica) y, por otro, de las relaciones entre la organización y agentes externos (principalmente, clientes y proveedores). A los distintos tipos de aprendizaje estudiados con anterioridad por la teoría evolutiva, como learning by doing ('aprender haciendo', en las actividades productivas habituales, resolviendo problemas) y learning by using ('aprender sobre la base del uso' y adaptación de nuevos sistemas técnicos), Lundvall añade el learning by interacting, tanto interno a la organización (sobre la base del trabajo en equipos multidisciplinares) como externo a la misma, en especial en las relaciones productor-usuario, típicas, por ejemplo, de la relación entre fabricantes especializados y grandes clientes.

Así, este autor habla de dos «modos de innovación», el basado en la ciencia y la tecnología (modo STI, Science, Technology and Innovation) y el basado en «hacer, usar e interactuar» (modo DUI, Doing, Using and Interacting) (Jensen et alii, 2007; Lundvall y Lorenz, 2007). El modo de innovación STI, típico de sectores de alta tecnología, se caracteriza por la importancia del conocimiento codificado, del personal científico interno y de las relaciones con instituciones científicotecnológicas externas. Por contra, en el modelo DUI prima la interacción entre personas y entre departamentos, así como el intercambio de conocimiento tácito. La relación estrecha con usuarios o clientes es otra característica típica de este modelo. 


\section{Sistema regional de innovación y pymes}

Además del modelo DUI o interactivo de innovación, un segundo segmento de la literatura relacionado especialmente con este trabajo, dentro de la perspectiva de los sistemas de innovación, es el de los sistemas regionales y, especialmente, los estudios recientes sobre la influencia del entorno regional en la actividad innovadora de las pymes.

Dentro del enfoque del sistema de innovación, definido en un primer momento como sistema nacional, la aplicación a escala regional ha alcanzado una notable aceptación en los últimos años (Braczyk et alii, 1996; Cooke y Morgan, 1998; Storper, 1997; Maskell y Malmberg, 1999; Cooke, Gómez y Etxeberria, 1997; Maskell, 2001; OECD, 2001). Estos autores contemplan la innovación como un proceso localizado, en el que factores contextuales específicos pueden promover los procesos de creación y aplicación de conocimiento. Se considera que la aglomeración de empresas supone un ahorro en los costes de transacción en las relaciones entre empresas y favorece las interacciones basadas en la confianza mutua y el intercambio de conocimiento no codificado. Se parte de la premisa de que las redes formales e informales entre agentes (empresas, gobierno, agentes de $\mathrm{I}+\mathrm{D}$, otros agentes del entorno), en un contexto de confianza, permiten minimizar los costes de transacción, facilitando el intercambio de conocimientos tácitos de carácter innovador. Las características institucionales de una región y sus infraestructuras de conocimiento se consideran importantes condiciones básicas que pueden servir de estímulo para promover las actividades innovadoras.

Se han realizado diferentes estudios que se centran en la influencia del entorno regional en la actividad innovadora de las pymes desde la perspectiva del sistema regional de innovación, cuestión que nos interesa especialmente en este trabajo. Estos estudios ofrecen conclusiones interesantes sobre la especificidad de los procesos de innovación en estas organizaciones, las fuentes externas y las relaciones de cooperación que establecen las empresas en sus procesos de innovación, así como la influencia del entorno regional en sus procesos de innovación.

Estos trabajos resaltan que, en la mayoría de las pymes, la innovación es un proceso que se asienta principalmente sobre bases internas de conocimiento (Freel, 2003; Freel y Harrison, 2006; Gebauer et alii, 2005; Kaufman y Tödtling, 2000). Las pymes poseen una base limitada de recursos, lo cual limita sus posibilidades a la hora de realizar actividades de $\mathrm{I}+\mathrm{D}$ y de establecer relaciones de cooperación con agentes externos. Entre las razones de estas limitaciones se encuentran la falta de recursos financieros y de personal, la falta de tiempo, la falta de know how tecnológico y la falta de capacidades para la búsqueda y selección de información relevante del exterior (Kaufmann y Tödtling, 2002; Smallbone et 
alii, 2003). En este contexto, se subraya la importancia de que las empresas hayan desarrollado competencias internas como condición indispensable para que puedan establecer relaciones fructíferas de cooperación con agentes externos y sean capaces de hacer uso de información externa e integrarla con la procedente del interior de la empresa (Freel y Harrison, 2006; Kauffman y Tödtling, 2000; Koschatzky y Sternberg, 2000).

Dada su menor capacidad para moldear e influenciar el entorno externo, la innovación se produce de manera reactiva, como respuesta a circunstancias cambiantes del entorno, y no forma parte de una estrategia proactiva a largo plazo (Freel, 2000; Hassink, 1997; Smallbone et al., 2003). Este carácter reactivo y a corto plazo de la innovación en pymes dificulta la cooperación con agentes regionales de $\mathrm{I}+\mathrm{D}$ (universidades, centros tecnológicos), los cuales configuran su agenda de investigación a medio o largo plazo (Hassink, 1997). La innovación en pymes presenta un marcado carácter market pull (Grotz y Braun, 1997; Doloreux, 2003, Kaufman y Tödtling, 2002). Los estudios coinciden en señalar el papel tractor del cliente en los procesos de innovación de las pymes y cómo, en numerosas ocasiones, éstas se ven impulsadas por sus clientes a realizar nuevos desarrollos tecnológicos (Hassink, 1997; Kauffman y Tödtling, 2002, 2003; Gebauer et alii, 2005).

Los estudios realizados desde el prisma del sistema regional de innovación prestan especial atención al análisis de las relaciones de cooperación que establecen las pymes en sus procesos de innovación, así como al ámbito geográfico en que éstas se circunscriben. El hecho de que la mayoría de las innovaciones sea market pull tiene como consecuencia que las redes de cooperación se limiten, para numerosas pymes, a relaciones con agentes dentro de la cadena de valor, clientes y proveedores (Freel, 2000, 2003; Doloreux, 2003). Como señalan Kaufman y Tödtling (2002), unas pocas relaciones duraderas y selectivas con estos agentes moldean la mayor parte de las actividades de innovación de las pymes. Esto es especialmente cierto para empresas que realizan innovaciones incrementales en sectores maduros, pero también para empresas con desarrollos basados en avances científicos (Freel, 2003; Hassink, 1997). Estas relaciones presentan, de manera frecuente, un carácter informal (Fritsch, 2001) y, en ellas, el precio no es el único factor determinante en una operación comercial. La existencia de valores compartidos, normas comunes y confianza entre las partes resultan de vital importancia (Doloreux, 2003, 2004; Asheim y Coenen, 2005; Hassink, 1997; Grotz y Braun, 1997).

Se señala que, fuera de estas relaciones que se producen en la cadena de valor, las pymes presentan escasos vínculos con empresas competidoras y agentes 
de I+D (Freel, 2000; Kaufmann y Tödtling, 2002; Gebauer et alii, 2005; Doloreux 2003, 2004; Koschatzky y Sternberg, 2000; Vickers y North, 2000; Grotz y Braun, 1997). Desde estos estudios se ha prestado especial atención a la relación con agentes de $\mathrm{I}+\mathrm{D}$, promovidos por las políticas de $\mathrm{I}+\mathrm{D}$ (políticas de creación de una oferta tecnológica) desde los años ochenta del pasado siglo. Los estudios realizados coinciden en apuntar que las estructuras de $\mathrm{I}+\mathrm{D}$ poseen un impacto limitado en los procesos de innovación en pymes.

Las investigaciones internacionales sugieren que la cooperación con los institutos de $\mathrm{I}+\mathrm{D}$ se circunscribe a empresas más grandes $\mathrm{y} / \mathrm{o}$ con mayores capacidades tecnológicas (Koschatzky y Zenker, 1999; Kaufmann y Tödtling, 2002). Hassink (1997) destaca que las empresas con mayores debilidades en tecnología, que debieran ser el grupo objetivo de estos agentes, tienden a ignorar estas infraestructuras de $\mathrm{I}+\mathrm{D}$, mientras que las empresas con mayores capacidades en $\mathrm{I}+\mathrm{D}$ e innovación son las que recurren a dichas infraestructuras en mayor medida. Como se ha mencionado, el carácter reactivo de las innovaciones en pymes dificulta la colaboración con agentes de $\mathrm{I}+\mathrm{D}$, que poseen horizontes de investigación a más largo plazo.

Estos estudios destacan también la existencia de factores, por parte de la oferta tecnológica, que dificultan estas relaciones. Se señala que los agentes de I+D no llegan a satisfacer la demanda de las empresas y que realizan una escasa contribución a resolver problemas específicos de éstas, así como en la aplicación de nuevas tecnologías en sus procesos de producción y nuevos productos (Gebauer et alii, 2005). Estos estudios resaltan que las empresas buscan en los centros tecnológicos información tecnológica básica, apoyo telemático y servicios de bajo valor añadido, e indican la necesidad de que estas infraestructuras se alineen en mayor medida con las necesidades específicas de las pymes (Asheim y Coenen, 2005; Vickers y North, 2000; Gebauer et alii, 2005).

Así mismo, también se señala la necesidad de que los agentes de $\mathrm{I}+\mathrm{D}$ presenten una estrategia más proactiva (Kaufman y Tödtling, 2002; Gebauer et alii, 2005; Vickers y North, 2000), y de que la transferencia de tecnología de estas infraestructuras de I+D se relacione con otras áreas de las empresas (planificación, marketing, finanzas, recursos humanos) (Vickers y North, 2000; Hassink, 1997).

A pesar de las limitaciones de las denominadas "políticas de oferta» que ponen de manifiesto estos estudios, se enfatiza en que la existencia de un entorno regional que favorezca la actividad innovadora resulta especialmente apropiado en el caso de las pymes, ya que éstas se encuentran más imbricadas en el entorno regional que las empresas más grandes (Fritsch, 2001; Koschatzky y Sternberg, 2000; Koschatzky y Zenker, 1999; Kauffmann y Tödtling, 2002). En este senti- 
do, Copus et alii (2008) concluyen que las diferencias interregionales en las tasas de innovación son la consecuencia de heterogeneidad regional y no de características observables de las empresas.

Estos estudios destacan que la región es especialmente importante para el intercambio de conocimiento tácito y para la provisión de mano de obra cualificada, mecanismos ambos muy importantes en los procesos de innovación de las pequeñas empresas (Asheim y Coenen, 2005; Kauffmann y Tödtling, 2003; Grotz y Braun, 1997; Doloreux 2003; Gebauer et alii, 2005).

No obstante, no todos los entornos regionales favorecen la actividad innovadora de las empresas. Algunos déficits de los sistemas regionales que pueden dificultar la actividad innovadora de las empresas son la falta de actores regionales relevantes (organizational thinness), la fragmentación o la falta de colaboración en la innovación entre agentes de la región y el lock in institucional social y cultural (Asheim e Isaksen, 2003; Kaufmann y Wagner, 2005; Tödtling y Trippl, 2005; Iammarino, 2005). Estos déficits se producen en mayor medida en regiones periféricas, donde prevalecen los sectores industriales tradicionales.

Para evitar el lock in se resalta la importancia de los vínculos extrarregionales (Fritsch, 2001; Koschatzky y Sternberg, 2000; Kauffmann y Tödtling, 2002, 2003; Arndt y Sternberg, 2000; Oinas, 2000). Los estudios señalan que la proximidad es más importante para la cooperación con agentes de I+D que para la cooperación dentro de la cadena de valor, donde alcanzan una notable relevancia las relaciones con clientes y proveedores internacionales (Fritsch, 2001; Doloreux 2003, 2004; Koscahtzky y Zenker, 1999; Gebauer et alii, 2005). En este sentido, Simmie (2002) resalta la importancia de combinar vínculos con clientes de carácter internacional con el conocimiento local por parte de proveedores, universidades y agentes de transferencia locales.

\section{Metodología del estudio empírico e hipótesis}

El trabajo empírico realizado toma como universo poblacional a las pymes industriales de la provincia española de Guipúzcoa. Para la recogida de datos se han combinado técnicas cualitativas (entrevistas en profundidad) con técnicas cuantitativas (encuesta telefónica) ${ }^{1}$.

1 Las entrevistas en profundidad tuvieron lugar a lo largo del primer semestre de 2007 y las mantuvieron los miembros del equipo de investigación con directores de Oficinas Técnicas, directores de I+D y gerentes de las citadas empresas, con una duración media de 75 minutos. Las empresas entrevistadas se encuadran en las siguientes áreas de actividad: 15 en fabricación de máquinas-herramienta; 11 son pymes con producto propio (industria química, industria de maquinaria de elevación, industria de transformación de la madera, materiales para la construcción, etc.); y las 21 restantes son empresas auxiliares (automoción, máquinas-herramienta, aeroespacial y elevación, fundamentalmente). Respecto al tamaño, se caracterizan por tener entre cincuenta y 
Las hipótesis principales que se pretenden contrastar por medio de la triangulación de evidencia cualitativa y cuantitativa son las siguientes:

1. La actividad innovadora de las pymes es de tipo incremental, con predominio del conocimiento tácito. Dicha actividad consiste en nuevas (para la empresa) combinaciones de conocimiento existente, relacionadas con frecuencia con la incorporación y adaptación de nuevos (para la empresa) componentes tecnológicos.

2. La capacidad interna de innovación de las empresas (relacionada con su tamaño y con la existencia de una unidad organizativa dedicada a innovación o I+D) influye en gran medida en sus patrones de cooperación.

3. Los procesos de innovación se derivan de un conjunto reducido de relaciones, principalmente con agentes de la cadena de valor (clientes y proveedores), donde los agentes de $\mathrm{I}+\mathrm{D}$ (como centros tecnológicos o universidades) juegan un papel secundario.

4. La proximidad geográfica y el sistema regional facilitan los procesos de innovación en las pymes industriales.

\section{Resultados}

\subsection{Caracterización de la actividad de innovación en las pymes industriales: incremental, basada en el conocimiento tácito y en la combinación nueva de conocimientos existentes}

La perspectiva de los sistemas de innovación subraya que la creación de nuevo conocimiento se basa normalmente en nuevas combinaciones de conocimiento existente. Se trataría, por tanto, de una innovación incremental, referida al desarrollo, mejora y modificación de productos y procesos, con una importante componente de conocimiento tácito. Esta caracterización de la innovación aparece reflejada en el estudio cualitativo realizado (adjuntamos algunas referencias significativas).

\footnotetext{
doscientos empleados, salvo en la industria auxiliar, donde el tamaño se reduce, en algunos casos, hasta los 15 empleados.

La encuesta telefónica se realizó en noviembre de 2008. Los informantes fueron los responsables de innovación de las pymes industriales: directores gerentes, directores de $\mathrm{I}+\mathrm{D}$ o directores de oficinas técnicas. Las empresas fueron seleccionadas de manera aleatoria entre una población total de 454 empresas industriales de 25 o más empleados radicadas en la provincia de Guipúzcoa y estratificadas por tamaño (Base de datos SABI de Bureau Van Dick, 2008). La muestra quedó definitivamente constituida por 147 empresas que cumplimentaron válidamente el cuestionario, con un error de muestra de $\pm 6,6 \%$ en estimaciones para un NC $95 \%$ y en el supuesto de variabilidad más desfavorable $(\mathrm{p}=\mathrm{q}=0,5)$.
} 
Nosotros no tenemos una vocación de inventar [...] Nosotros tiramos de lo que hay en el mercado, hacemos un desarrollo del problema que tiene el cliente e intentamos encontrar la herramienta o la solución más adecuada. Nosotros no inventamos, bastante tenemos con fabricar la máquina a medida del cliente. (E3)

No se trata de mandar cohetes a la luna. Muchas veces nosotros tenemos un producto maduro; puedes decir que está poco sujeto a cambios drásticos y radicales, y digamos que la tecnología que le puedes aplicar pues también es bastante limitada, ¿no? [...] Entonces, son pequeñas cosas y que muchas veces, pues eso, ¿qué misterio tienen? [... ]. Pero, al final, estás innovando. Es una innovación de cara al mercado. (E38)

El tema es que hay que innovar pero, más o menos, porque lo pide el cliente. $Y$, a veces, innovas en cosas que no se escriben, pero tú sabes que has mejorado: en la manera de servirle, o en la logística que le estás aplicando, o porque has cambiado o... ¿no? (E37)

Las pymes con producto propio consideran la innovación como una tarea de captación, adaptación, desarrollo e implementación, en sus procesos y productos, de tecnologías que se encuentran en el mercado. Serían, por tanto, en gran medida, «integradoras» de nuevas tecnologías. Por otro lado, las innovaciones que se acometen en las empresas de la industria auxiliar están relacionadas, normalmente, con mejoras en los procesos y/o implantación de nuevas máquinas que posibiliten la reducción de costes y la consecución de aumentos en la productividad, en orden al cumplimiento rentable de las estipulaciones que han planteado los clientes (generalmente, plazos, calidades y precios). Así las cosas, las actividades internas de $\mathrm{I}+\mathrm{D}$ «formal» (o $\mathrm{I}+\mathrm{D}$ en sentido estricto) desempeñan un papel residual para la mayor parte de las organizaciones que componen la muestra analizada.

En relación con el tipo de innovación efectuada por las pymes industriales, de manera consistente con el análisis cualitativo, el estudio cuantitativo sugiere que las innovaciones en producto $(54,9 \%)$ y proceso $(40,7 \%)$ copan los proyectos realizados en cooperación por parte de las empresas. Solo un 1,1\% manifiesta haber innovado en organización recientemente y un 3,3\% en mercados. Dentro de este contexto, son las pymes de mayor tamaño las que efectúan innovación en producto, mientras que las de menor tamaño focalizan sus esfuerzos hacia el proceso.

Un aspecto subrayado por el modelo de Aalborg es el aprendizaje interactivo interno a la organización, referido tanto a las relaciones entre los actores que componen la empresa (dirección, ingenieros, trabajadores) como a la interacción entre áreas (por ejemplo, entre diseño, producción y marketing). 
Tabla 1. Contenido del proyecto en el que se ha cooperado con un agente externo (\%)

\begin{tabular}{|c|c|c|c|}
\hline $\mathrm{N}=93$ & $\begin{array}{c}<50 \\
\text { empleados }\end{array}$ & $\begin{array}{c}\geq 50 \\
\text { empleados }\end{array}$ & Total \\
\hline Producto & 53,7 & 56,8 & 54,9 \\
\hline Proceso & 42,6 & 37,8 & 40,7 \\
\hline Organización & 0 & 2,7 & 1,1 \\
\hline Mercados & 3,7 & 2,7 & 3,3 \\
\hline
\end{tabular}

Cabe afirmar que, cuanto más pequeña es la empresa, más tienden a estar centralizadas las decisiones sobre las innovaciones y menor es el papel de los empleados en la implantación de las mismas. Son los miembros del Consejo de Administración y/o Dirección los que asumen la adopción de decisiones en este ámbito. Como una gran parte del colectivo de empresas de menor tamaño vive al día, los empleados centran su jornada en sacar adelante el trabajo y resolver problemas relacionados con las operaciones, de tal manera que no se les suelen presentar oportunidades de participación, ni tienen tiempo para mucho más.

No obstante, y de manera paulatina, la participación de los trabajadores es cada vez más visible en los procesos de innovación de algunas empresas. Por tanto, aunque todavía no se trata de prácticas extendidas, en la mayoría de las pymes entrevistadas existe una creciente interacción entre los actores en relación con los procesos de innovación.

Cada $\mathrm{X}$ tiempo nos juntamos con la gente y bueno oye, «¿qué cosas veis que se pueden mejorar, qué cosas no?»» «Las que son viables adelante, las que no, pues no se puede, por esto y por eso». Pretendemos, de alguna forma, que participe todo el mundo en mayor o menor medida, que participe todo el mundo, desde la dirección hasta el último operario, con mejor o peor éxito, pero es lo que hay, puedes preguntar a cualquiera, mucho del personal está motivado por eso. (E9)

Este año hemos dado otro salto en este aspecto y hemos hecho el Plan de Gestión entre 25 personas [...]. El cambio con respecto a hace tres años es que yo (Director Técnico) definía todo y ahora les estoy pasando a ellos [...]. Mejor que eso (el procedimiento para la innovación) es que el equipo sea multidisciplinar, que el equipo aporte. Más que perder el tiempo en descargar y poner allí (en el depositario informático contemplado en el procedimiento) las herramientas y los ficheros, lo relevante es que el equipo esté realmente motivado, esté aportando ideas, y coja el tema con ganas [...]. Lo que hacemos es trabajar en equipo y que cada uno cuente sus ideas. (E24) 
Sí, son participativos, en eso tenemos una empresa... [...]. Muchas veces lo desarrolla la persona que va a hacer luego el montaje, que es la persona que más experiencia tiene y es el que toma partido y dice yo haría esto así, así o así o lo colocaría así. ¿'Te acuerdas del material que colocamos en aquella máquina? Pues, nos vendría bien aquí también'. Sí que toman parte y tienen un cierto contacto con los proveedores. (E12)

Como también sucede, con el objetivo de añadir valor al cliente e intentar implicar al mayor número de personas de la organización, en diferentes departamentos y niveles, en la adopción de las decisiones. Hemos apreciado una palpable inquietud en un creciente grupo de empresas por compartir a escala organizacional los retos de la innovación.

Sí, sí. Se hacen presentación de proyectos, hacemos brainstorming también para ver posibilidades de mejora [... ]. Luego cuando se desarrolla el proyecto también en los AMFES, el análisis de modos de fallos [...] se necesita gente de producción, gente de ingeniería, gente de montaje y gente comercial. (E10)

Desde el momento en el que en la Dirección ya se ha discutido y se ha planteado el tema concreto a desarrollar, se abre un pedido interno y la fase de estudio de su viabilidad. Se abre una reunión multidisciplinar donde se escucha a los diferentes departamentos. Luego se abre una rueda de información y de captura de otros departamentos que nos puedan aportar más información sobre lo que ya se estaba haciendo, sobre las deficiencias observadas, etc. (E25)

Nos vienen muchas propuestas y, por eso, el año pasado decidimos crear estos equipos de mejora en cuanto hay algún tipo de propuesta; bien porque hay algún diseño que es un poco innovador y en vez de tomar soluciones solamente los de arriba, los diseñadores, siempre tenemos una persona de mecanizado, por lo menos, otra de soldadura, otro del afilado, de diferentes secciones por las que vayan a pasar estas herramientas, para que aporten ideas, dificultades si se diseña de una manera o de otra a la hora de mecanizar, entonces sí que hay... nosotros por suerte tenemos una plantilla bastante dinámica y creo que nos aportan bastantes ideas. (E17)

No obstante, las reticencias que muestran algunos trabajadores ante las dinámicas de participación, así como las dificultades a la hora de mantener la tensión organizativa necesaria para sostener un sistema de gestión participativa, dan una idea de la dificultad de implantar cambios organizativos de esta naturaleza que favorezcan la innovación. 
Como en todas las organizaciones, hay personas que hasta se resisten. (E16)

La participación de los trabajadores en la mayoría de los casos es importante, si bien, no suele ser fácil. La motivación de la gente [...] no suele ser fácil. (E20)

Sí, sí, lo que pasa es que cuesta porque hemos pasado de un modelo un poco jerárquico, poco dinámico, a un modelo que exige participar mucho, y cuesta, y eso lleva tiempo. Nosotros con el tema este desde hace tres años ya andábamos dándole más vueltas y hay momentos que ves que has evolucionado, pero luego te vas parando y la gente se va quemando, porque eso requiere mucho esfuerzo de las personas y hay que darle otro cambio de giro y tal. (E24)

Sí, un poco lo que os he comentado antes. Sobre todo lo hemos notado después del cambio generacional, los jóvenes son más dinámicos. Una persona de 50 años para arriba es, en general [... ] viene más a meter las horas y punto. Los chavales son más dinámicos, están haciendo las cosas y están también proponiéndote mejoras constantemente, no son conformistas «aunque esto se ha hecho así toda la vida, esto se puede hacer mejor».(E17)

\subsection{Influencia de la capacidad interna de innovar en los patro- nes de cooperación: tamaño y estructura}

Los estudios realizados bajo el prisma del sistema de innovación resaltan la importancia de las bases internas de conocimiento en los procesos de innovación de las pymes. Se subraya la importancia de que las empresas hayan desarrollado competencias internas como condición indispensable para que puedan establecer relaciones fructíferas de cooperación con agentes externos y sean capaces de hacer uso de información externa e integrarla con la procedente del interior de la empresa.

En el estudio cualitativo, se han analizado, además de la variable tamaño, algunos aspectos organizativos internos, como la formalización de las herramientas de Dirección ( p. e., la planificación estratégica), que inciden en la actividad innovadora y en los patrones de cooperación de las pymes industriales. A su vez, dentro de la encuesta realizada, las variables que reflejan en mayor medida la capacidad interna de innovar que tiene la empresa son el tamaño y la existencia de una estructura para la innovación (oficina técnica y/o departamento de I+D).

En el estudio cualitativo hemos podido constatar el papel que juega la Oficina Técnica en los procesos de innovación de las pymes industriales (en particular, en aquellas con menor tamaño), en tanto que estructura para la innovación. El papel de esta unidad está centrado en satisfacer las necesidades del día a día (estudio de 
pedidos, presupuestación, desarrollo de máquinas, adaptación de procesos productivos, relación con proveedores, etc.), que compatibiliza, no sin tensiones, con actividades a medio y largo plazo como vigilancia tecnológica y asistencia a ferias. Esta oficina técnica, sin embargo, se encuentra, en general, alejada de las actividades de $\mathrm{I}+\mathrm{D}$ formal (proyectos de $\mathrm{I}+\mathrm{D}$ en cooperación, prospección tecnológica, patentes y otras actividades a más largo plazo).

Nuestro departamento de Innovación es la Oficina Técnica. Lo que es el trabajo de todos los días hay que hacerlo y, de vez en cuando, mirar un poco hacia adelante para ver un poco hacia dónde vamos. La capacidad, al final, es limitada en una empresa de 100 tíos. Lo que es evidente es que, si en cinco años no haces nada, en menos, en tres años no haces nada, te has colado [...]. (E30)

El problema que veo a las empresas pequeñas como ésta, es que en la Oficina Técnica los técnicos hacemos todos los lanzamientos de producto, hacemos todos los diseños necesarios del herramental necesario para estampación, realizamos las compras y apoyamos la fabricación [...] Con dos personas tenemos que hacer todos los desarrollos. Además, nuestra función principal es presentar al cliente un montón de ofertas, ofertar, estudiarlo, los precios, labor comercial, el comercial da el precio, llegar a un acuerdo, hacer el lanzamiento de ese producto [...] Estamos prácticamente viviendo el día a día, constantemente [...]. (E2)

Diez personas aquí arriba (en la Oficina Técnica) y otras tres personas (en planta). Su misión principal es la de establecer nuevos procesos. Hay parte que están en Producción, pero gran parte del tiempo están trabajando en nuevos procesos. (E26)

En el estudio cualitativo se ha podido también comprobar que la variable 'tamaño' incide en aspectos de planificación y organización que, a su vez, inciden de manera positiva en la actividad innovadora de las pymes. Se constata que la planificación estratégica está relacionada con el tamaño empresarial y que son las pymes de mayor tamaño las más avanzadas en estas prácticas de gestión. Así mismo, hemos podido detectar que la planificación estratégica se vincula efectivamente con actividades de $\mathrm{I}+\mathrm{D}$ formal (generación de estructura para $\mathrm{I}+\mathrm{D}$, planificación tecnológica, planes de producto, patentes, prospección tecnológica, participación en proyectos de $\mathrm{I}+\mathrm{D}$ en cooperación, etc.). Este hecho sugiere que la $\mathrm{I}+\mathrm{D}$ interna está positivamente relacionada con los niveles de planificación de la empresa.

La falta de planificación en la gestión de la actividad innovadora de las empresas afecta de manera negativa a la colaboración con agentes de I+D (fundamentalmente, centros tecnológicos y universidades) y a la concurrencia a convocato- 
rias de financiación de actividades de innovación, que requieren que las empresas realicen una planificación estratégica a medio y largo plazo de sus actividades de innovación.

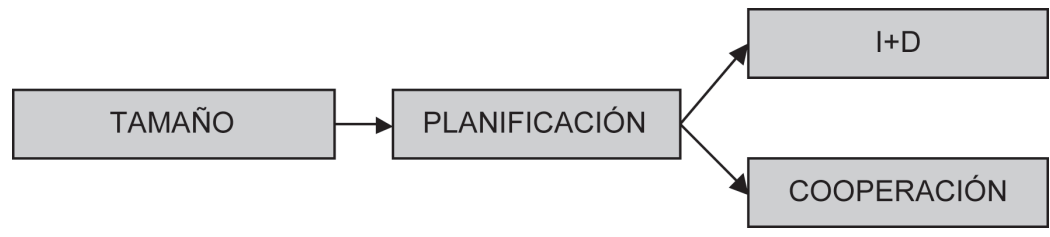

En consonancia con los resultados del estudio cualitativo, dentro de la encuesta la variable 'tamaño' muestra una relación importante con muchos de los aspectos de las actividades de innovación y, por supuesto, de cooperación. Como mínimo, el desarrollo de actividad innovadora es más palpable cuanto mayor es el tamaño de la empresa. Además, la existencia de una estructura para la innovación está claramente relacionada con el tamaño empresarial.

Tabla 2. Empresas innovadoras y estructura para la innovación en cooperación según tamaño

\begin{tabular}{|l|c|c|c|}
\hline N=147 & $\begin{array}{c}<50 \\
\text { empleados }\end{array}$ & $\begin{array}{c}\geq 50 \\
\text { empleados }\end{array}$ & Total \\
\hline Empresas que innovan (\%) & 85,7 & 92,6 & 87,8 \\
\hline Existencia de oficina técnica (\%) & 76,1 & 79,6 & 76,9 \\
\hline Existencia de departamento de I+D (\%) & 30,8 & 64,8 & 43,5 \\
\hline
\end{tabular}

Como hemos visto, la realización de actividades de innovación de las pymes industriales se canaliza, en gran medida, a través de la oficina técnica. La existencia de un departamento de $\mathrm{I}+\mathrm{D}$ es menos frecuente y se circunscribe a las pymes más grandes. Un 64,8\% de las empresas con 50 o más empleados que fueron encuestadas cuentan con este tipo de unidad, frente al 30,8 \% entre las empresas de menor tamaño (véase la Tabla 2).

La existencia de una estructura para la innovación también influye en el establecimiento de relaciones con agentes externos. Las empresas que disponen de una unidad para la actividad innovadora cooperan significativamente en mayor medida con agentes externos en materia de innovación. Un 72\% de las empresas que cuentan con oficina técnica y/o departamento de $\mathrm{I}+\mathrm{D}$ cooperan con agentes externos en innovación, mientras que entre las empresas que no disponen de estructura para estas actividades, la gran mayoría $(71,4 \%)$ no coopera con agentes externos. 
Gráfico 1 . Relación entre estructura y propensión a la cooperación $(\mathrm{N}=147)$

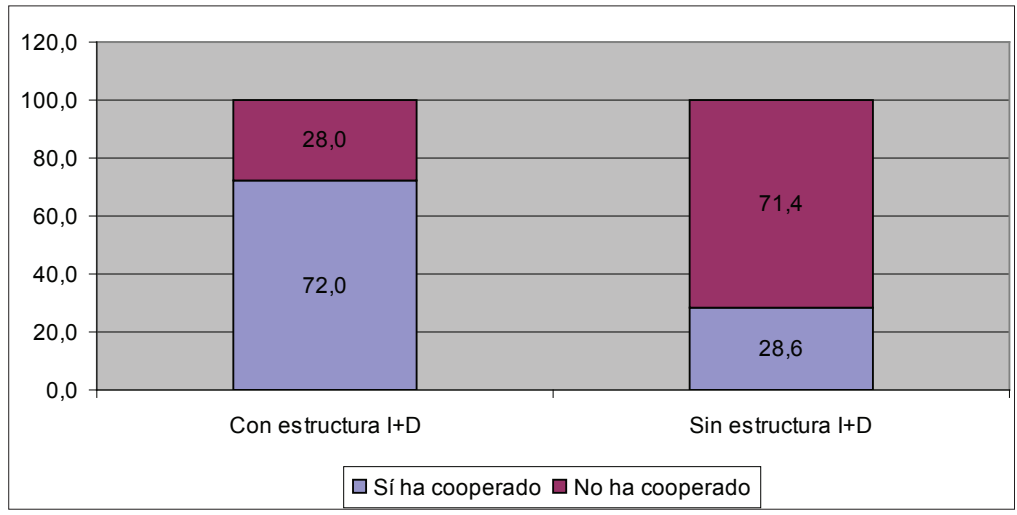

\subsection{Innovación como proceso de aprendizaje interactivo: confianza y reciprocidad en una relación duradera con un reducido grupo de agentes externos}

De acuerdo con la teoría relativa a los Sistemas de Innovación, los procesos de aprendizaje interactivo que establecen las empresas con agentes externos se basan, en gran medida, en relaciones de confianza necesarias para el intercambio de conocimiento tácito, no codificado.

Lundvall (1992b) utiliza el concepto de «mercado organizado» para referirse a este fenómeno. Frente al carácter anónimo (y con una función de mera transmisión de información codificada) del mercado en la teoría neoclásica, los mercados organizados se caracterizarían por el intercambio de información cualitativa, la cooperación y la confianza mutua.

Hemos trabajado con clientes en algunos desarrollos de nuevos productos [...] Colaboramos con clientes, ellos nos daban sus ideas, aportaban en las primeras reuniones de diseño e incluso en algún cliente hemos hecho las pruebas de validación de producto, las hemos hecho en casa del cliente. Con algún cliente estuvimos durante un año, hicimos unos equipos de mejoras conjuntos. (E17)

Luego suele haber unos acuerdos con unos buenos clientes [...] Hay una forma en la cual en el primer año de desarrollo en casa del cliente, pues tienes un feedback con él, de cosas que le ocurren a él, cosas que pediría él, ya más al detalle [...] y al final, tú a este producto has llegado [... tras haber mantenido una relación durante unos años. (E6) 
El mismo proveedor del robot, incluso hemos conseguido que nos dejasen algún robot para trabajar con él a nivel de formación. El tema este de la visión, un proveedor de visión artificial [...] Echamos mano, según el tema que sea, de lo que haga falta. (E9)

Con algunos proveedores tenemos también muy, muy buena relación. Mucha confianza [...] Nos ha tocado muchas veces que a un comercial le han cambiado de zona, se ha cambiado de zona de distribución y hemos peleado con la casa matriz. «Déjamelo porque llevo 10 años trabajando con él, sabe las necesidades, sabe qué productos, y el consejo que me da él, va a misa» y hemos peleado y ha habido fabricantes finales que nos lo han permitido. (E12)

Por otro lado, en nuestro estudio cuantitativo se pone de manifiesto que, si bien la cooperación formalizada en un proyecto conjunto es el tipo de cooperación que alcanza mayor relevancia dentro de la cooperación para la innovación, aspectos como el intercambio organizado de información y experiencias, el establecimiento de contactos informales para la adquisición de la información y los usos pilotos de innovaciones ( $\mathrm{p}$. e., maquinaria) poseen también cierta importancia en las cooperaciones efectuadas por las pymes, pudiéndose colegir que elementos como la confianza y la reciprocidad son singularmente importantes en las relaciones de intercambio que establecen las empresas; tanto más cuanto menor es el tamaño de las empresas.

\section{Gráfico 2. Tipo de cooperación con partners empresariales $(\mathrm{N}=50)$}

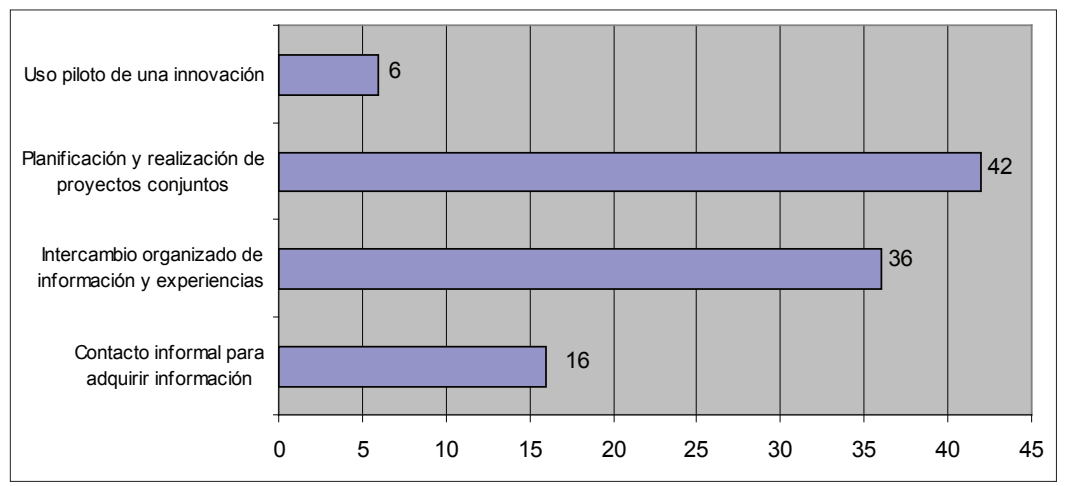

La aparición de estos elementos de reciprocidad requiere la generación de relaciones selectivas de carácter estable y duradero con un número reducido de actores. En este sentido, en los estudios realizados a escala internacional sobre pymes en entornos regionales, se resalta que los procesos de innovación en estas 
empresas se derivan de un conjunto reducido de relaciones, principalmente con agentes dentro de la cadena de valor (clientes y proveedores).

Las relaciones de cooperación para la innovación son tanto más frecuentes cuanto mayor es el tamaño de las empresas: un 70,4\% de las pymes con 50 o más empleados coopera con agentes externos en sus procesos de innovación, porcentaje que desciende al 60,4\% en las pymes de menos de 50 empleados. Además, la variedad de agentes externos con los que cooperan las pymes aumenta también con el tamaño (véase Tabla 3).

Tabla 3. Cooperación en innovación y variedad de partners de cooperación (\%)

\begin{tabular}{|l|c|c|c|}
\hline & $\begin{array}{c}<50 \\
\text { empleados }\end{array}$ & $\begin{array}{c}\geq 50 \\
\text { empleados }\end{array}$ & Total \\
\hline $\begin{array}{l}\text { Empresas que han cooperado con agentes externos } \\
\text { en proyectos de innovación (\%) (N=147) }\end{array}$ & 60,4 & 70,4 & 63,3 \\
\hline Variedad de partners de cooperación (\%) (N=93) & & & \\
\hline 1 o 2 agentes diferentes & 40,0 & 31,6 & 36,6 \\
\hline 3 o 4 agentes diferentes & 36,4 & 39,5 & 37,7 \\
\hline 5 o más agentes diferentes & 23,7 & 29,0 & 25,8 \\
\hline
\end{tabular}

La encuesta realizada a pymes industriales confirma que las pymes cooperan con un grupo reducido de agentes externos. Así, más de la mitad de las empresas encuestadas ha colaborado como máximo con tres tipos diferentes de agentes. Estas relaciones son, además, duraderas en el tiempo, como se puede deducir de las respuestas de las empresas a la pregunta sobre la regularidad de la cooperación. Un 43,3\% de las empresas manifiesta haber cooperado más de tres veces con el mismo agente con el que desarrolló últimamente un proyecto de innovación relevante, siendo un 21,1\% las empresas que han cooperado 2 o 3 veces con el mismo agente y un 35,6\% las que lo han hecho por primera vez (véase el gráfico 3).

Para las pymes encuestadas resulta especialmente relevante la cooperación dentro del ámbito de confianza desarrollado en la cadena de valor (el 61,3\% dice haber cooperado con clientes y el $53,8 \%$ con proveedores en proyectos de innovación), así como en el ámbito de las relaciones que se producen entre las empresas del propio grupo (38,7\%). Como se puede deducir de los datos reflejados en la siguiente tabla, el impacto de las estructuras para la $\mathrm{I}+\mathrm{D}$ (centros tecnológicos, universidades y CIC) es más discreto. 
Gráfico 3. Regularidad de cooperación con el partner con el que se ha colaborado en proyecto de innovación $(\mathrm{N}=93)$

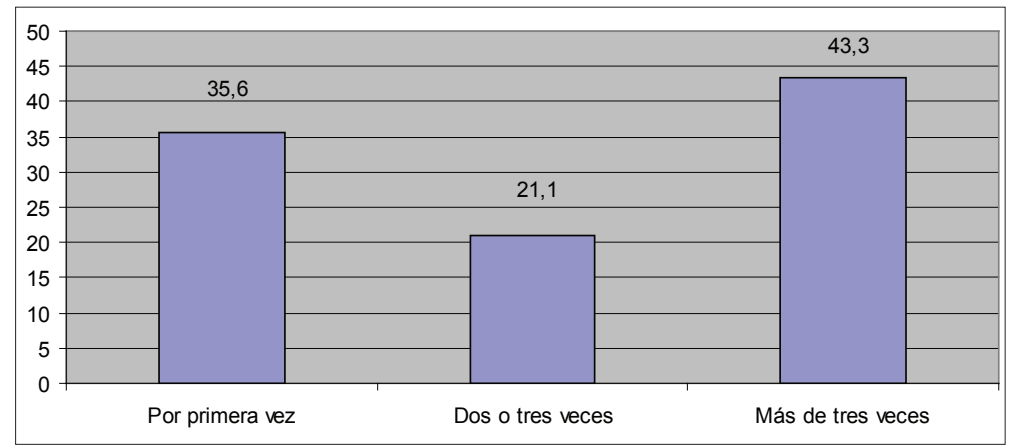

Llaman la atención, no obstante, tres hechos: el peso de las ingenierías y consultorías privadas; el relativamente alto impacto de los centros tecnológicos, sobre todo si se compara con el bajo peso atribuido por la literatura internacional; $y$ la aparición de la colaboración en innovación de un actor no considerado por los estudios sobre innovación hasta el momento, como son los centros de FP.

Gráfico 4. Partners para la cooperación en proyectos de innovación (\%) (N=93)

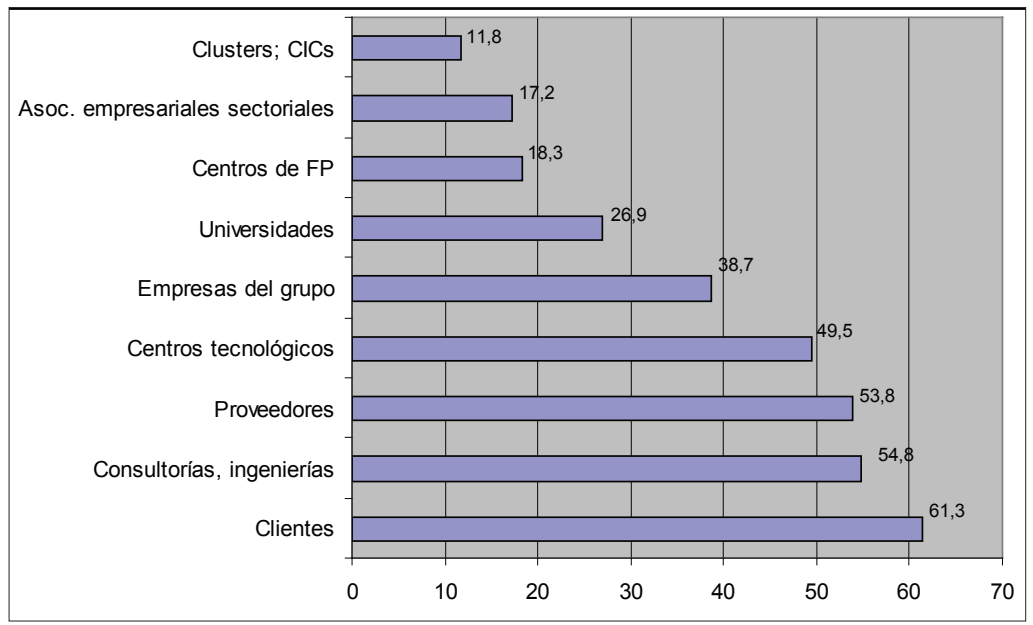

Las ingenierías contribuyen a efectuar desarrollos tecnológicos para las pymes, caracterizándose por ser capaces de dar respuesta a los requerimientos de las empresas en cortos plazos de tiempo a un coste razonable. Las consultoras en gestión de la innovación, por su parte, realizan una labor de vigilancia tecnológica, análisis de patentes, prospectiva tecnológica y apoyan a las empresas en la ela- 
boración de sus planes estratégicos. Estas empresas ofrecen un apoyo importante para la consecución de proyectos dentro de programas de financiación pública.

En relación con el peso relativamente alto de los centros tecnológicos como agente de innovación, cabe señalar que, como se ha comentado anteriormente, han constituido uno de los pilares de las políticas regionales del departamento de Industria del Gobierno vasco durante los últimos 25 años. Esta política intensiva ha hecho que sea palpable su influencia sobre las empresas. Su elevado número, los ámbitos de especialización — ligados en cierta medida con las necesidades de algunos sectores representativos de la industria-, el tamaño y la vinculación a las empresas vía cofinanciación a través de los programas públicos hacen posible que su impacto en las pymes del País Vasco sea considerable.

Los centros de formación profesional han irrumpido en el sistema de innovación cubriendo el nicho de mercado de servicios tecnológicos que los centros tecnológicos, ingenierías y universidad no alcanzan a satisfacer: el formado por las pymes de menor tamaño. Los principales factores que hacen posible su aparición como interlocutor de las empresas para la innovación son los siguientes: cuentan con un notable know how tecnológico aplicado; un profesorado reciclado gracias a la formación continua a empresas que también imparten, además de la reglada; una maquinaria e instalaciones que la gran mayoría de pymes no se pueden costear; una amplia cartera de empresas que, en muchos casos, excede a la propia comarca en la que se radican; y una vocación de dar servicio tecnológico a las empresas. En el estudio cualitativo hemos podido detectar cómo estos centros, además de formación reglada y no reglada, realizan algunos servicios de consultoría y de índole tecnológica que apoyan los procesos de innovación de las empresas pequeñas.

IMH, por su conocimiento de lo que es mecanizado, de herramienta, nos ha ayudado muchísimo con el tema de laboratorios, metrología, pruebas, en ese aspecto, nos han ayudado y nos están ayudando muchísimo en la parte del metal. (E17)

De hecho incluso hay centros con los cuales tenemos un acuerdo de colaboración (p. e. con la escuela del Goierri) y, bueno, estamos haciendo trabajos con ellos y les hemos dado accesos a bases de datos nuestras, locales y [...] a nivel global también. (E50)

Entonces, se planteó una auditoría de motivación y de las personas, y de ahí sí salió una gran necesidad de participación. (E28) 


\subsection{Proximidad geográfica y sistema regional}

La perspectiva de los sistemas regionales de innovación resalta la importancia de la proximidad geográfica en los procesos de intercambio de conocimiento tácito. La existencia de un mercado de trabajo cualificado, la tradición de cultura industrial, la existencia de redes de clientes y proveedores y la competencia e imitación entre las empresas producen procesos de clusterización y especialización territorial. Además, de acuerdo con la literatura de sistemas regionales, la existencia de un entorno regional que favorezca la actividad innovadora resulta especialmente importante en el caso de las pymes, ya que éstas se encuentran más imbricadas en el ámbito regional que las empresas más grandes.

La encuesta realizada confirma la importancia de la proximidad geográfica. Un 48,9\% de las relaciones de cooperación de las empresas encuestadas lo son con agentes ubicados en el territorio histórico de Guipúzcoa. El porcentaje aumenta hasta el 68\% si consideramos las relaciones dentro de la Comunidad Autónoma del País Vasco (véase Tabla 4). Cuando la cooperación se realiza con empresas, el porcentaje de cooperación regional es el 50\%, mientras que, de acuerdo con la literatura internacional analizada, en el caso de la cooperación con agentes de I+D, la proximidad geográfica juega todavía un papel más importante (90,5\%).

Tabla 4. Ubicación del partner de cooperación según su naturaleza (\%)

\begin{tabular}{|l|c|c|c|}
\hline$N=93$ & Empresa & Agente I+D & Total \\
\hline Provincia & 40,0 & 59,5 & 48,9 \\
\hline Región & 10,0 & 31 & 19,6 \\
\hline Estado & 26,0 & 7,1 & 17,4 \\
\hline UE & 20,0 & 2,4 & 12,0 \\
\hline Fuera de la UE & 4,0 & 0 & 2,2 \\
\hline Total & 100 & 100 & 100 \\
\hline
\end{tabular}

La perspectiva del sistema regional de innovación incide en la importancia del entorno institucional y, en particular, de las políticas públicas. La puesta en marcha de instrumentos de financiación pública de actividades de innovación favorece la innovación en pymes. En la CAPV, como hemos visto anteriormente, estas políticas han favorecido también la cooperación entre pymes y centros tecnológicos.

Según la encuesta realizada, un 43,2\% de las empresas ha contado con financiación pública para la realización del proyecto de innovación más importante llevado a cabo en los últimos tres años. En las entrevistas cualitativas realizadas las empresas consideran de manera positiva la existencia de estas ayudas públicas. 
Las subvenciones ayudan a superar el riesgo e incertidumbre asociados a estos proyectos. Además, las empresas consideran necesarios estos programas para poder disfrutar de un $\mathrm{I}+\mathrm{D}$ competitivo que les permita una menor repercusión en los costes de los productos o servicios que desarrollan para sus clientes. No obstante, también ven aspectos mejorables en la gestión de estos programas, como son una excesiva burocracia y trámites asociados, así como el que se considere que se discrimina a las empresas pequeñas frente a las más grandes.

Las pymes más grandes acceden en mayor medida a programas de financiación pública. Un 37,7\% de las pymes más pequeñas han contado con ayuda pública para la relación del proyecto de innovación más importante de los últimos tres años, frente al 62,9\% de las pymes con 50 o más empleados (véase el gráfico 5).

\section{Gráfico 5. Financiación pública del proyecto según tamaño} y naturaleza del partner (\%) ( $\mathrm{N}=93)$

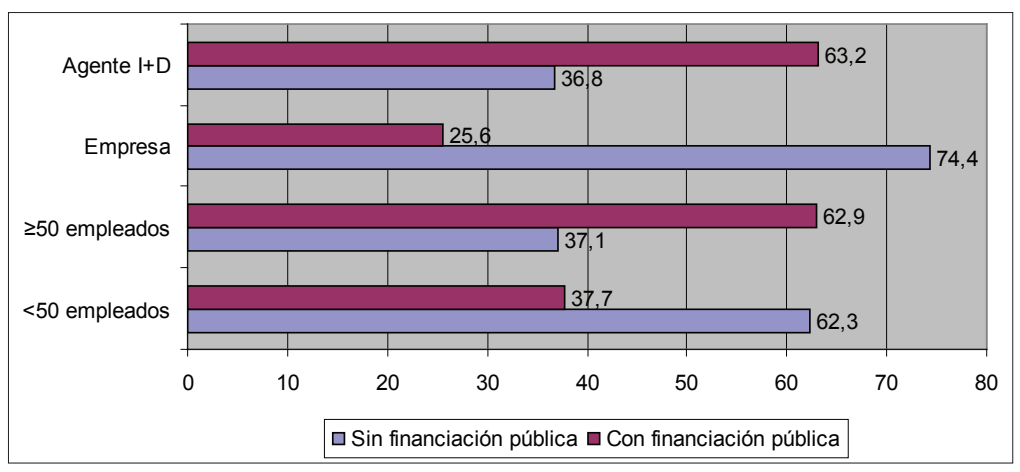

En el estudio cualitativo hemos podido constatar que estas ayudas, en general, favorecen a las empresas de mayor tamaño y/o mayores capacidades de innovación. La documentación que exigen estos programas requiere un cierto grado de planificación y formalización de las actividades de innovación y, por ello, el escaso nivel de planificación existente en las pymes incide de manera negativa en el acceso a programas de financiación pública. Las empresas más pequeñas y con menor capacidad de innovación quedan fuera de los programas públicos de ayudas, muchas veces por desconocimiento, pero también porque estas ayudas, debido a la formalización y documentación que requieren, se adecuan mejor a empresas más grandes, que han estructurado en cierto grado su actividad de innovación.

La sensación que tenemos nosotros es que hacemos bastante innovación y no lo plasmamos. Por otra parte, tampoco cobramos las subvenciones [...] Porque nos falta, digamos, plasmar eso [...] ese mecanismo nuevo [...] Entonces, no lo 
sabemos explotar, y el problema es que, a nivel de subvención, hace falta tener como un volumen importante. Que se puedan cuantificar en ese valor y que quizás no encontramos la forma de acceder [... a las subvenciones. Y al no tener ese acicate, digamos, pues tampoco se documentan (los proyectos) [...] Bueno, es un poco el déficit que tenemos. Entonces, estamos intentando ver cómo agrupar diversos proyectos de tipo similar para conseguir acceder (a las subvenciones). (E44)

La colaboración con centros tecnológicos incide de manera positiva en la obtención de financiación pública. Un 63,2\% de las cooperaciones con agentes de $\mathrm{I}+\mathrm{D}$ han recibido financiación pública, mientras que en el caso de cooperación con empresas este porcentaje se reduce al 25,6\% (véase el gráfico 5). En el estudio cualitativo hemos comprobado que, en buena medida, la colaboración con estos agentes se realiza en el marco de programas públicos de ayuda a la $\mathrm{I}+\mathrm{D}$. Los centros tecnológicos prestan un apoyo indispensable para la gestión de la documentación de estos proyectos.

Los centros de FP son, en general, bien valorados como partners de cooperación, produciéndose un aumento de la valoración de este agente a medida que disminuye el tamaño de la empresa (véase la Tabla 5). Este dato apunta a la importancia que los centros de formación profesional pueden tener en los procesos de innovación de las pymes, sobre todo de las empresas con menos recursos para ello, como son, en particular, las pequeñas y muy pequeñas empresas. Creemos que la potencialidad de este agente no ha sido todavía suficientemente reconocida por parte de las políticas de I+D. En el estudio cualitativo hemos podido detectar cómo estos centros, además de formación reglada y no reglada, realizan algunos servicios de consultoría y de índole tecnológica que apoyan los procesos de innovación de las empresas pequeñas.

Tabla 5. Valoración media ${ }^{1}$ de los partners de cooperación según tamaño

\begin{tabular}{|l|c|c|c|}
\hline \multicolumn{1}{|c|}{$\mathrm{N}=93$} & $\begin{array}{c}<50 \\
\text { empleados }\end{array}$ & $\begin{array}{c}\geq 50 \\
\text { empleados }\end{array}$ & Total \\
\hline Empresas de su mismo grupo & 3,72 & 3,89 & 3,81 \\
\hline $\begin{array}{l}\text { Proveedores de equipos, material, componentes o } \\
\text { software }\end{array}$ & 3,57 & 3,8 & 3,67 \\
\hline Clientes & 3,62 & 3,68 & 3,64 \\
\hline $\begin{array}{l}\text { Consultorías, ingenierías y/u otras empresas de } \\
\text { servicios }\end{array}$ & 3,72 & 3,57 & 3,66 \\
\hline Universidades & 3,38 & 3,18 & 3,29 \\
\hline Centros tecnológicos & 3,48 & 3,72 & 3,61 \\
\hline
\end{tabular}




\begin{tabular}{|l|c|c|c|}
\hline Centros de FP & 3,92 & 3,75 & 3,88 \\
\hline $\begin{array}{l}\text { Clusters y/o centros de investigación cooperativa } \\
\text { (CICs) }\end{array}$ & 3,6 & 3,83 & 3,73 \\
\hline Asociaciones empresariales sectoriales & 3,56 & 4,2 & 3,79 \\
\hline
\end{tabular}

${ }^{1}$ Valoraciones de 1 a 5

\section{Conclusiones}

En este trabajo hemos mostrado que la innovación en las pymes industriales analizadas es de tipo incremental y consiste, principalmente, en la integración de nuevos componentes técnicos en los productos de las empresas, así como, en general, en la mejora gradual de procesos y productos. Los procesos de innovación consisten, por tanto, en la búsqueda y adaptación de nuevas combinaciones de conocimiento existente. En dichos procesos prima el conocimiento tácito, el learning by doing ('aprender haciendo', esto es, a partir de la resolución de problemas relacionados con las actividades productivas habituales) y el learning by using ('aprender utilizando', integrando y modificando nuevas tecnologías que surgen en el mercado).

La tercera «pata» del modelo DUI de Aalborg (learning by interacting) aparece más desarrollada en su aspecto externo que en su aspecto interno. En el interior de las pymes, el modelo de gestión y toma de decisiones tiende a ser jerárquico y centralizado. En general, la relación entre áreas se limita al ámbito directivo y la participación de los trabajadores está bastante limitada, si bien se ha comprobado la existencia de un creciente número de experiencias en las que la interacción entre los diferentes niveles y funciones de la empresa redunda en mejoras en los procesos de innovación. Respecto al aprendizaje interactivo externo, es más habitual la colaboración con unos pocos clientes y proveedores. Esta colaboración, especialmente con los clientes, tiene una importancia crítica para los procesos de innovación de las pymes industriales. Se trata de relaciones basadas en elementos de confianza y reciprocidad, continuadas en el tiempo. Las pymes analizadas demuestran una gran capacidad para «aprender» tecnológicamente con los clientes y adaptarse a sus necesidades.

Por otro lado, destaca también el nivel de cooperación con los centros tecnológicos, mayor de lo esperado a la luz de la literatura internacional reciente, y la importancia de las ayudas públicas, que alcanzan a un número importante de empresas. Si bien la labor de los centros tecnológicos parece dirigirse en buena medida a la ayuda en la solución de problemas o servicios concretos, comienzan a 
observarse colaboraciones de mayor entidad y más continuadas. En todo caso, el papel de estos agentes, principal instrumento creado por las políticas regionales, es más importante que en otros países o regiones.

La importancia del entorno regional ha quedado patente, con evidencia tanto cuantitativa (procedencia de los partners de cooperación) como cualitativa. La proximidad geográfica y la «densidad regional» son compatibles con un elevado nivel de apertura al exterior (como por ejemplo en el sector de máquina-herramienta, representado en la muestra del estudio cualitativo).

En suma, creemos que el modelo interactivo, dentro de la perspectiva de los sistemas de innovación, resulta especialmente adecuado para el análisis de los procesos de innovación en las pymes industriales. A nuestro entender, este modelo permite corregir un cierto sesgo que existe todavía en los estudios regionales, según el cual el problema de la innovación consistiría en una suerte de «transferencia» desde el «subsistema de generación de conocimiento» (los agentes de I + D) hacia el «sistema de aplicación de conocimiento» (las empresas). Siguiendo a Lundvall y otros, hay que partir de la premisa de que el agente innovador (generador de conocimiento con valor económico) es la empresa. En sus procesos de búsqueda, a partir de las capacidades internas existentes y las peticiones de los clientes, las pymes industriales muestran una notable capacidad de aprendizaje interactivo externo, así como de integración y desarrollo de tecnologías. Como asignatura pendiente respecto a modelos similares exitosos como el danés, cabe mencionar las dinámicas de aprendizaje interno entre los agentes sociales que componen la empresa, manifiestamente mejorables. Finalmente, respecto a las relaciones con los agentes externos de $\mathrm{I}+\mathrm{D}$, es cierto que las pymes deben reforzar sus capacidades de innovación y su demanda hacia dichos agentes. Pero también lo es que estos agentes, así como las políticas regionales que se implementan, pueden mejorar su acoplamiento con el tipo de innovación existente en las empresas, al mismo tiempo que contribuyen a elevar el nivel tecnológico de las mismas.

\section{Bibliografía}

Arndt, O. y Sternberg, R. (2000). «Do Manufacturing Firms Profit from Intraregional Innovation Linkages? An Empirical Based Answer», en European Planning Studies, n.* 8 (4): 465-485.

Asheim, B. T. y Coenen, L. (2005). «Knowledge bases and regional innovation systems: comparing nordic clusters», en Research Policy, $\mathrm{n}_{+}^{\circ} 34$ (8): 1731190 . 
Asheim, B. T. e Isaksen, A. (2003). «SMEs and the regional dimension of innovation», en Asheim, B. T.; Isaksen, A., Nauwelaers, C. y Tödtling, F. (eds.), Regional innovation policy for small-medium enterprises. Cheltenham: Edwar Elgar, págs. 21-46.

Braczy , H.; Cooke, P. y Heidenreich, R. (eds.) (1996). Regional Innovation Systems. The Role of Governances in a Globalized World. London: University College London Press.

Cooke, P; Gomez, M. y Etxebarria, G. (1997). «Regional Innovation Systems: Institutional and Organizational Dimensions», en Research Policy, n. ${ }^{\circ}$ $26(4-5) \div 475-491$.

Cooke, P. y Morgan, K. (1998). The associational economy: Firms, regions and innovation, Oxford: Oxford University Press.

Copus, A.; Skuras, D. y Tsegenidi, K. (2008). «Innovation and peripherality: an empirical comparative study of SMEs in six european union member countries», en Economic Geography, n+ ${ }^{\circ} 84$ (1): 51-82.

Doloreux, D. (2003). «Regional innovation systems in the periphery: the case of Beauce in Quebec (Canada)», en International Journal of innovation management, n* $7(1) \div 67-94$.

Doloreux, D. (2004). «Regional innovation systems in Canada: a comparative study», en Regional Studies, n+ 38 (5): 479-492.

Dosi, G.; Freeman, C.; Nelson, R. R.; Silverberg, G. y Soete, L. (eds.) (1988). Technological Change and Economic Theory. London: Pinter.

Edquist, C. (1997). Systems of Innovation: Technologies, Institutions, and Organizations. London; Pinter.

Freel, M. S. (2000). «Strategy and structure in innovative manufacturing SMEs: the case of an English Region», en Small Business Economics, n* 15 (1): 27-45.

Freel, M. S. (2003). «Sectorial patterns of small firm innovation, networking and proximity», en Research Policy, n. 32 (5): 751-770.

Freel, M. S. y Harrison, R. T. (2006). "Innovation and cooperation in the small firm sector: Evidence from 'Northern Britain'», en Regional Studies, n. ${ }^{\circ}$ $40(4): 289-305$.

Freeman, C. (1987). Technology, Policy, and Economic Performance: Lessons from Japan. London, Pinter Publishers.

Fritsch, M. (2001)+ «Co-operation in regional innovation systems», en Regional Studies, n+ $35(4) \div 297-307$. 
Gebauer, A.; Woon Nam, C. y Parsche, R. (2005). «Regional technology policy and factors shaping local innovation networks in small German cities», en European Planning Studies, n. ${ }^{\circ}$ 13(5): 661-683.

Grotz, R. y Braun, B. (1997). «Territorial or transnational networking: spatial aspects of technology oriented cooperation within the German mechanical Engineering Industry», en Regional Studies, n. 31 (6): 545-557.

Hassink, R. (1997). «Technology transfer infrastructures: some lessons from experiences in Europe, the US and Japan», en European Planning Studies, n. ${ }^{\circ}$ $5(3): 351-370$.

IAmmarino, S. (2005). «An evolutionary integrated view of regional systems of innovation: concepts, measures and historical perspectives», en European Planning Studies, n+ ${ }^{\circ} 13$ (4): 497-518.

Jensen, M. B.; Johnson, B.; Lorenz, E. y Lundvall, B. A. (2007). «Forms of knowledge and modes of innovation», en Research Policy, vol. 36, n. ${ }^{\circ}$, págs. 680-693.

Kaufmann, A. y Tödtling, F. (2000). «Systems of innovation in traditional industrial regions: the case of Styria in a comparative perspective», en Regional Studies, n. 34: 29-40.

Kaufmann, A. y Tödtling, F. (2002). «How effective is innovation support for SMEs? An analysis of the region of Upper Austria», en Technovation, $\mathrm{n}^{\circ} 22$ (3): 147-159.

Kaufmann, A. y Todtling, F. (2003). «Innovation pattern of SMEs», en Asheim, B.; Isaksen, A.; Nauwelaers, C. y Tödtling, F. (eds.). Regional innovation policy for small-medium enterprises. Cheltenham: Edwar Elgar, págs. 78-115.

Kaufmann, A. y Wagner, P. (2005). «EU regional policy and the stimulation of innovation: the role of European Regional Development Fund in the objective 1 Region Burgenland», en European Planning Studies, n. 13 (4): 581-599.

Koschatzky, K. y Sternberg, R. (2000). «R\&D cooperation in innovation systems- some lessons from the European Regional Innovation Survey (ERIS)», en European Planning Studies, n. ${ }^{\circ} 8$ (4): 487-471.

Kosckatzky, K. y Zenker, A. (1999). "The regional embeddedness of small manufacturing and service firms: regional networking as knowledge source for innovation?», en Working Papers Firms and Regions N. ${ }^{\circ}$ R2/1999, Fraunhofer Institute Systems and Innovation Research.

Lundvall, B. A. (1992a). National Systems of Innovation: Towards a Theory of Innovation and Interactive Learning. London: Pinter. 
Lundvall, B. A. (1992b). «User-producer relationships, national sytems of innovation and internationalization», en Lundvall, B. (ed.), National systems of innovation. London: Pinter, págs. 45-67.

Lundvall, B. A. y Lorenz, E. (2007). «Modes of Innovation and Knowledge Taxonomies in the Learning economy», CAS workshop on Innovation in Firms, Oslo.

Maskell, P. (2001). «Social capital, innovation and competitiveness», en BAron, S.; Field, J. y Schuller, T. (eds.), Social Capital: Critical perspectives. Oxford: Oxford University Press, págs. 111-123.

Maskell, P. y Malmberg, A. (1999). «Localised learning and industrial competitiveness», en Cambridge Journal of Economics, n. ${ }^{\circ} 23$ (2): 167-186.

Nelson, R.R. (ed.) (1993). National Innovation Systems: A Comparative Analysis. Oxford: Oxford University Press.

OECD (2001). The Well-being of Nations, The Role of Human and Social Capital. Paris: OECD.

Oinas, P. (2000). «Distance and learningः does proximity matter?», en BoeKema, F.; Morgan, K.; Bakkers, S. y Rutten, R. (eds.), Knowledge, innovation and economic growth. The theory and practice of learning regions. Cheltenham: Edwar Elgar, págs. 57-59.

Simmie, J. (2002). «Knowledge spillovers and reasons for the concentration of innovative SMEs», en Urban Studies, n. ${ }^{\circ} 39$ (5-6): 885-902.

Smallbone, D.; North, D. y Vickers, I. (2003). «The role and characteristics of SMEs», en Asheim, B.T.; Isaksen, A.; Nauwelaers, C. y Tödthing, F. (eds.), Regional innovation policy for small-medium enterprises. Cheltenham: Edwar Elgar, págs. 3-20.

Storper, M. (1997). The regional economy. New York, Guilford Press.

Suárez Sánchez-Ocaña, A. (CEO Ocio Networks). «Innnovando, que es gerundio», en Diario de Navarra, 29 de noviembre de 2009, pág. 14.

Todtling, F. y Trippl, M. (2005). «One size fits all? Towards a differenciated regional innovation policy approach», en Research Policy, n. 34 (8): $1203-$ 1219.

Vickers, I. y North, D. (2000). «Regional technology Initiatives: Some insights from the English Regions», en European Planning Studies, n. ${ }^{\circ} 8$ (3): 301-318. 